\title{
BEAM DYNAMICS DESIGN AND SIMULATION STUDIES OF THE APT SUPERCONDUCTING LINAC
}

\author{
T. P. Wangler, B. Blind, S. Nath, R. D. Ryne, and K. R. Crandall* \\ Los Alamos National Laboratory, Los Alamos, NM 87545 USA \\ *Techsource, Santa Fe, NM 87594-1057
}

\section{APT LINAC}

The 100-mA Accelerator for Production of Tritium (APT) nominal design[1],[2] consists of a normal-conducting proton linac to 211-MeV, followed by a 700-MHz, cw superconducting linac to $1030 \mathrm{MeV}$. The APT superconducting linac accelerates the beam using two distinct 5-cell elliptical superconducting-cavity shapes, designed with cell lengths corresponding to two different geometric beta values $\beta_{\mathrm{G}}=0.64$ and 0.82 . A summary of the main parameters is shown in Table 1 , and the beamdynamics parameters are given in Table 2.

Table 1. APT Superconducting Linac Parameters

\begin{tabular}{|l|l|l|l|}
\hline Parameter & $\begin{array}{l}\beta_{\mathbf{G}}=\mathbf{0 . 6 4} \\
\text { section 1a }\end{array}$ & $\begin{array}{l}\beta_{\mathbf{G}}=\mathbf{0 . 6 4} \\
\text { section 1b }\end{array}$ & $\begin{array}{l}\beta_{\mathbf{G}}=\mathbf{0 . 8 2} \\
\text { section 2 }\end{array}$ \\
\hline Input energy & 211 & 242 & 471 \\
\hline$\beta_{\mathrm{MIN}}$ & 0.578 & 0.607 & 0.746 \\
\hline$\beta_{\mathrm{MAX}}$ & 0.607 & 0.746 & 0.879 \\
\hline $\mathrm{E}_{\mathrm{A}}(\mathrm{MV} / \mathrm{m})$ & 6.1 to 5.4 & 5.1 to 5.3 & 7.1 to 5.3 \\
\hline $\mathrm{E}_{\mathrm{PK}} / \mathrm{E}_{\mathrm{A}}$ & 3.12 & 3.12 & 2.77 \\
\hline $\mathrm{B}_{\mathrm{PK}} / \mathrm{E}_{\mathrm{A}}(\mathrm{G} / \mathrm{MV} / \mathrm{m})$ & 69.3 & 69.3 & 62.7 \\
\hline Bore radius $(\mathrm{mm})$ & 65 & 65 & 80 \\
\hline Cavs./klystron & 2 & 3 & 2 \\
\hline Cavs./cryomod. & 2 & 3 & 4 \\
\hline No. cryomods. & 6 & 30 & 35 \\
\hline $\mathrm{P}_{\mathrm{BEAM}} /$ cav. $(\mathrm{MW})$ & 0.28 & 0.28 & 0.42 \\
\hline $\mathrm{P}_{\text {COUPLER }}(\mathrm{MW})$ & 0.14 & 0.14 & 0.21 \\
\hline
\end{tabular}

\section{LINAC PARAMETER ISSUES}

Our design approach takes advantage of the large velocity acceptance of the superconducting cavities. As a guide to an initial layout, an analytic model of multi-cell cavities excited in a $\pi$-mode was used to provide an expression for the transit-time factor $\mathrm{T}$ as a function of particle velocity. A simple cavity-field distribution was assumed where the fields are uniform in the accelerating gaps and zero immediately outside the gaps. For a cavity with $\mathrm{N}$ identical cells, $\mathrm{T}$ can be expressed as a product of two separate factors $T=T_{G} T_{S}$. The gap factor $T_{G}$, which is also the transit-time factor for a single gap of length $\mathrm{g}$, RF wavelength $\lambda$, and particle-velocity $\beta$, is given by the expression $\mathrm{T}_{\mathrm{G}}=\sin (\pi \mathrm{g} / \beta \lambda) /(\pi \mathrm{g} / \beta \lambda)$. The synchronism factor $\mathrm{T}_{\mathrm{S}}$ is a function of $\mathrm{N}$ and of the ratio of the local velocity, $\beta$, to the cavity geometric velocity, $\beta_{\mathrm{G}}=2 \mathrm{~L} / \lambda$, where $\mathrm{L}$ is the cell length. The synchronism factor is given by:

$$
\mathrm{T}_{\mathrm{S}}=\left\{\begin{array}{l}
(-1)^{\frac{\mathrm{N}-1}{2}} \cos \left(\mathrm{N} \pi \beta_{\mathrm{G}} / 2 \beta\right) / \mathrm{N} \cos \left(\pi \beta_{\mathrm{G}} / 2 \beta\right), \mathrm{N} \text { odd } \\
(-1)^{\frac{\mathrm{N}}{2}+1} \sin \left(\mathrm{N} \pi \beta_{\mathrm{G}} / 2 \beta\right) / \mathrm{N} \cos \left(\pi \beta_{\mathrm{G}} / 2 \beta\right), \mathrm{N} \text { even }
\end{array}\right\} \text { (1) }
$$

Equation 1 can be compared with direct numerical computation of $\mathrm{T}$ for the real cell shape. We find that the velocity dependence in the model is modified when penetration of the fields into the beam pipe on the end cells is included. For the two 5-cell APT elliptical cavities, the model gives a good fit to the numerical results when we choose $\mathrm{g}=0.463 \beta_{\mathrm{G}} \lambda$ in the expression for $\mathrm{T}_{\mathrm{G}}$, and we replace $\beta_{\mathrm{G}}$ by $1.04 \beta_{\mathrm{G}}$ in Eq. 1 .

Table 2. Beam Dynamics Parameters*

\begin{tabular}{|c|c|c|c|}
\hline Parameter & $\begin{array}{l}\beta_{G}=0.64 \\
\text { sect. } 1 \mathrm{a}\end{array}$ & $\begin{array}{l}\beta_{G}=0.64 \\
\text { sect. 1b }\end{array}$ & $\begin{array}{l}\beta_{G}=0.82 \\
\text { sect. } 2\end{array}$ \\
\hline Quad lattice type & Doublet & Doublet & Doublet \\
\hline Lattice period (m) & 4.88 & 6.18 & 8.54 \\
\hline Synch. phase (deg) & -30 to -35 & -30 to -42 & -30 \\
\hline $\begin{array}{l}\mathrm{I}=0 \text { transv. phase } \\
\text { adv./period (deg) }\end{array}$ & 78 to 63 & 80 to 58 & 81 to 72 \\
\hline $\begin{array}{l}\mathrm{I}=0 \text { long. phase } \\
\text { adv./period (deg) }\end{array}$ & 46 & 56 to 40 & 53 to 25 \\
\hline $\begin{array}{l}\text { Transverse tune- } \\
\text { depression ratio }\end{array}$ & $\begin{array}{l}0.43 \text { to } \\
0.37\end{array}$ & $\begin{array}{l}0.39 \text { to } \\
0.42\end{array}$ & $\begin{array}{l}0.44 \text { to } \\
0.62\end{array}$ \\
\hline $\begin{array}{l}\text { Longitudinal tune- } \\
\text { depression ratio }\end{array}$ & $\begin{array}{l}0.33 \text { to } \\
0.37\end{array}$ & $\begin{array}{l}0.36 \text { to } \\
0.40\end{array}$ & $\begin{array}{l}0.39 \text { to } \\
0.34\end{array}$ \\
\hline $\begin{array}{l}\text { Trans. rms norm. } \\
\text { emittance (mm-mrad) }\end{array}$ & $\begin{array}{l}0.23 \text { to } \\
0.24\end{array}$ & $\begin{array}{l}0.24 \text { to } \\
0.26\end{array}$ & $\begin{array}{l}0.26 \text { to } \\
0.25\end{array}$ \\
\hline $\begin{array}{l}\text { Long. rms norm. } \\
\text { emittance (deg-MeV) }\end{array}$ & $\begin{array}{l}0.38 \text { to } \\
0.36\end{array}$ & $\begin{array}{l}0.36 \text { to } \\
0.42\end{array}$ & $\begin{array}{l}0.42 \text { to } \\
0.58\end{array}$ \\
\hline $\begin{array}{l}\text { Rms phase width } \\
\text { (deg) }\end{array}$ & 4.1 to 2.9 & 3.5 to 2.4 & 2.5 to 2.1 \\
\hline $\begin{array}{l}\text { Aperture radius/max } \\
\text { rms beam size }\end{array}$ & 24 to 30 & 22 to 31 & 33 to 51 \\
\hline
\end{tabular}

* Values are quoted for zero machine errors. Including realistic machine errors typically doubles the emittances, and gives tune depressions greater than 0.5 .

For APT the RF power delivered to the beam greatly exceeds the power dissipated in the superconducting walls. Then, the rf power required per cavity, $\mathrm{P}_{\mathrm{C}}$, is approximately equal to the beam power, which is given as the product of the beam current times the particle energy gain $\Delta \mathrm{W}$ per cavity, as: 


$$
P_{C}=I \Delta W / q=I E_{A} \frac{T(\beta)}{T_{M A X}} \cos (\phi) N \beta_{G} \lambda / 2
$$

The beam current $\mathrm{I}$ is averaged over an $\mathrm{RF}$ period, $\mathrm{E}_{\mathrm{A}}$ is the accelerating gradient defined as the product of the spatial average of the axial accelerating field times the transit-time factor $\mathrm{T}_{\mathrm{MAX}}=\mathrm{T}\left(\beta_{\mathrm{T}}\right)$, where the velocity $\beta_{\mathrm{T}}$ gives the maximum transit-time factor. $T(\beta)$ is the transittime factor at the local velocity $\beta$ of the beam centroid, $\phi$ is the phase of the field when the beam centroid is at the center of a cavity, and $\mathrm{N}$ is the number of cells per cavity. The velocities, $\beta_{\mathrm{T}}$ and $\beta_{\mathrm{G}}$, are not exactly equal because of the gap factor $T_{G}$, which increases with increasing particle velocity.

Among the design parameters that must be chosen for each section (each section uses identical cavity shapes) of a proton superconducting linac include $\mathrm{N}, \beta_{\mathrm{G}}, \mathrm{E}_{\mathrm{A}}$ and $\phi$. One must also choose the number of sections, and the minimum and maximum velocities $\beta_{\text {MIN }}$ and $\beta_{\text {MAX }}$ for each section. These parameters must be chosen consistent with the design objectives, and for APT there were five goals. 1) Deliver the maximum usable power per klystron. 2) Maximize the number of cavities that are able to perform within the design specifications of accelerating gradient and peak surface fields. 3) Provide acceptable beam-dynamics performance. 4) Minimize the overall accelerator length. 5) Minimize the number of sections to reduce the overall cavity-development effort. These requirements cannot all be satisfied simultaneously. The best that can be done is to find an acceptable compromise.

In searching for a choice of $\beta_{\mathrm{G}}$ that satisfies the first and second criteria, we were initially influenced by the following considerations. From Eq.2, it can be seen that to maintain constant beam power for each cavity in a constant $-\beta_{\mathrm{G}}$ section, the value of $\mathrm{E}_{\mathrm{A}}$ can be chosen to compensate for the variations in $T(\beta)$. The largest values of $E_{A}$ and of the peak surface fields will be at the ends of each section where $T(\beta)$ is lowest. If the strategy to satisfy the second criterion is to minimize the highest peak surface field in a section, defined by fixed minimum and maximum velocity values $\beta_{\mathrm{MIN}}$ and $\beta_{\mathrm{MAX}}$, the optimum choice of $\beta_{\mathrm{G}}$ would be the value that provides equal values of T( $\beta)$ at both $\beta_{\mathrm{MIN}}$ and $\beta_{\mathrm{MAX}}$.

However, a better procedure for satisfying the second criterion is suggested by considering what happens to the accelerating gradient distribution over the same fixed velocity range if $\beta_{\mathrm{G}}$ is increased by a small amount. Because the cavities become longer, over most of the range the first-order effect is that for fixed input power the accelerating gradients and peak surface fields will decrease. An exception is at the low-velocity end of the section, where the gradients and peak fields will increase because of the decrease in transit-time factor. The result of a small increase in $\beta_{\mathrm{G}}$ is that most cavities will require lower gradient and peak surface fields, while a few cavities at the low-velocity end of the section will require higher values. If the objective of the second criterion was better achieved by reducing the average peak surface field rather than just the maximum values, a better strategy would be to increase $\beta_{\mathrm{G}}$.

To decide on the best strategy, we compared the required or design distribution of peak surface electric fields with an estimate of the expected peak surface field distribution (see Fig.1), based on the approximately three hundred $1500-\mathrm{MHz}$ superconducting cavities in the CEBAF linac. These data were reduced by an empirical field-emission factor, assumed to equal the ratio of surface areas to the minus one fourth power, and increased by a factor 1.3 to account for technology improvements since CEBAF was constructed.[3] We concluded that the strategy of a modest increase of $\beta_{\mathrm{G}}$ to reduce the average value of the peak surface field is a better approach, because it allows us to increase the number of cavities that can reach usable fields, resulting in fewer cavities that have to be rebuilt or sent back for reprocessing.

\section{APT LINAC PARAMETER CHOICES}

Assuming that the electromagnetic properties of the cavities have been determined so that $T(\beta)$ is known, we must choose values of $N, \beta_{\mathrm{MIN}} / \beta_{\mathrm{G}}$ and $\beta_{\mathrm{MAX}} / \beta_{\mathrm{G}}$ for each section. For APT we chose $\mathrm{N}=5$ as a trial value. To define the constant- $\beta_{\mathrm{G}}$ sections in a way that addresses the second criterion described above, we chose nonsymmetric values $\mathrm{T}\left(\beta_{\mathrm{MIN}} / \beta_{\mathrm{G}}\right)=0.7 \mathrm{~T}_{\mathrm{MAX}} \quad$ and $\mathrm{T}\left(\beta_{\mathrm{MAX}} / \beta_{\mathrm{G}}\right)=0.95 \mathrm{~T}_{\mathrm{MAX}}$, corresponding to $\beta_{\mathrm{MIN}} / \beta_{\mathrm{G}}=0.908$ and $\beta_{\mathrm{MAX}} / \beta_{\mathrm{G}}=1.174$. These choices allow us to cover the full velocity range with two constant- $\beta_{\mathrm{G}}$ sections, which satisfies the fifth criterion. The value of $\beta_{\mathrm{MIN}}=0.578$ for the first section, known as the medium-beta section (later divided into two subsections), corresponds to the output velocity of the normal-conducting linac. The value of $\beta_{\text {MIN }}$ for the second or high-beta section was chosen to equal $\beta_{\mathrm{MAX}}=0.747$ of the medium-beta section. The two resulting values of $\beta_{\mathrm{G}}$ are 0.64 and 0.82 , respectively.

To satisfy the first criterion, we want to use all the available RF power per klystron, which is $0.84 \mathrm{MW}$. Equation 2 may be used to choose parameter values that make use of all the power. For the medium-beta section, assuming trial values $\mathrm{N}=5$ and $\phi=-30^{\circ}$, we find that one klystron can deliver $0.28 \mathrm{MW}$ to three cavities if $\mathrm{E}_{\mathrm{A}}=4.72 \mathrm{MV} / \mathrm{m}$. For the high-beta cavities, assuming $\mathrm{N}=5$ and $\phi=-30 \mathrm{deg}$, Eq. 2 shows that one klystron can deliver $0.42 \mathrm{MW}$ to two cavities, if $\mathrm{E}_{\mathrm{A}}=5.52 \mathrm{MV} / \mathrm{m}$. The electromagnetic field-solver codes yield corresponding values of the peak surface fields for the two cavities, $14.7 \mathrm{MV} / \mathrm{m}$ and $15.3 \mathrm{MV} / \mathrm{m}$, respectively, for electric fields, and $346 \mathrm{G}$ and $327 \mathrm{G}$, respectively, for magnetic fields. These are conservative peak surface field values. Conservative power coupler values can be obtained by using two couplers per cavity. The values of $\phi$ are adjusted to provide optimum focusing and matching, as we discuss in the next section.

The choices for $\beta_{\mathrm{MIN}} / \beta_{\mathrm{G}}$ and $\beta_{\mathrm{MAX}} / \beta_{\mathrm{G}}$ produce a distribution of the peak surface field that is maximum at the low-velocity end of each section (see Fig. 1). The relatively small value of $\beta_{\mathrm{MAX}}$ allows us to switch at 
relatively low velocity from the medium-beta to the longer high-beta cavities, which improves the real-estate gradient and shortens the linac (fourth criterion).

The choice of $\mathrm{N}=5$ for APT led to practical values for accelerating gradients and peak surface fields. Generally, varying $\mathrm{N}$ causes several changes. A larger $\mathrm{N}$ reduces the velocity acceptance of the transit-time factor, as described by Eq.1. Additionally, for larger $\mathrm{N}$ at fixed beam power, the average accelerating gradient and peak surface electric field are reduced, but the longer cryomodule means that the average or real-estate gradient is also reduced, lengthening the linac. The longer cryomodule also increases the focusing period, which may increase the beam size. For APT we found no significant improvement from choices other than $\mathrm{N}=5$.

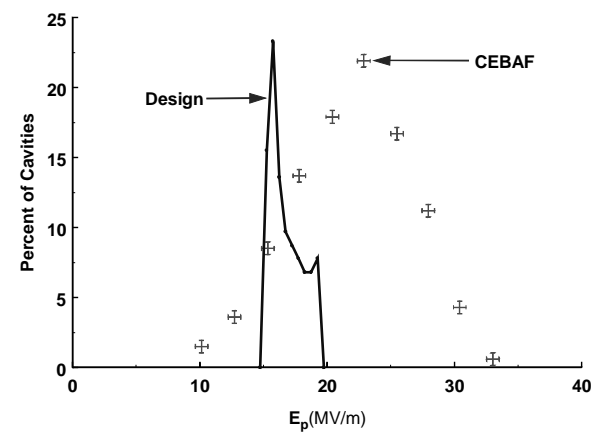

Figure 1: Comparison of the APT design and the scaled CEBAF experimental peak surface electric-field distributions.

\section{APT FOCUSING DESIGN}

The transverse focusing in APT is obtained using room-temperature quadrupole doublets installed between the cryomodules. This approach was preferred over the use of superconducting quadrupoles to simplify the cryomodule design. Doublets were favored over a FODO singlet lattice to reduce the focusing period and the maximum rms beam size. The medium-beta cryomodules in subsection $1 \mathrm{~b}$ contain three cavities driven by a single klystron. Each high-beta cryomodule in section 2 contains four cavities and uses two klystrons. To reduce the focusing period and provide better beam matching to the normal-conducting linac, which has a very short focusing period, we began with a medium-beta transition subsection (subsection 1a), consisting of six shorter 2cavity cryomodules.

Current-independent matching [4] was used at all focusing transitions, and is achieved by maintaining equal values of the zero-current phase-advance per unit length on each side of every focusing transition. The zero-current phase advance per focusing period after each transition was chosen near $80^{\circ}$ to provide the strongest transverse focusing, while staying safely below the envelope instability at $90^{\circ}$. At $1030 \mathrm{MeV}$, it was constrained to equal $72^{\circ}$ to provide a current-independent match to the high-energy transport line. The quadrupole gradients in the FODO lattice of the normal-conducting linac were decreased smoothly from 100 to $211 \mathrm{MeV}$ to help achieve the 211-MeV match. As a second step, small adjustments were applied to the gradients of the two quadrupoles on each side of that transition. The quadrupole gradients in each superconducting section were varied smoothly from beginning to end to accomplish the match into the next section.

With fixed accelerating gradients, longitudinal matching was achieved by adjusting the synchronous phase $\phi$ in the superconducting cavities, so that the longitudinal phase advance per unit length was equal on both sides of every focusing transition, while maintaining the constant energy gain $\Delta \mathrm{W}$ per cavity that is characteristic of each section. This results in equal values of $\Delta \mathrm{W} \tan (\phi) / \mathrm{D}$ on each side of a transition, where $\mathrm{D}$ is the period length. The values of $\phi$ were ramped smoothly in each section to achieve the matches.

Our choice of quadrupole gradients is not the only one we could have made. For example, we could have chosen the gradients to equipartition the beam in section 2 , but this choice had the disadvantage of weakening the transverse focusing and increasing the transverse beam sizes. The resulting tune-depression ratios, when machine errors are included, are well above a value of 0.4 , where chaos is observed in beam-halo models. Multiparticle simulation studies confirm that the design procedures provide good current-independent matches, and good overall beam-dynamics performance with a low risk of beam loss. Also, since the quadrupole gradients and phases are adjustable, experimental optimization is possible during the commissioning.

In spite of the large velocity acceptance of the cavities, one may wonder whether operation at velocities far from $\beta_{\mathrm{G}}$ might cause large longitudinal emittance growth, associated with a large cell-to-cell variation of the phase throughout the five cells of a cavity. For the APT design, we found that the maximum beam-phase slip between the first and fifth cells was about 100 degrees (for the $\beta_{\mathrm{G}}=0.82$ cavity at $471 \mathrm{MeV}$ ). Nevertheless, the beam-dynamics code showed no significant effect caused by this phase slip. The results of a detailed cell-by-cell treatment are indistinguishable from the simpler approach of calculating the dynamics using a single equivalent gap, using the phase of the middle cell.

\section{ACKNOWLEDGMENTS}

The authors acknowledge support from the U.S. Department of Energy. T.P.W. thanks F. Krawczyk for electromagnetic computations, and J. Delayen, $\mathrm{H}$. Padamsee, and R. L. Gluckstern for helpful discussions.

\section{REFERENCES}

[1] G. P. Lawrence and T. P. Wangler, Proc. 1997 Particle Accelerator Conf., Vancouver, B.C., Canada (May, 1997).

[2] G.P. Lawrence, XIX International Linear Accelerator Conf. Chicago, Ill. (August, 1998).

[3] These empirical factors were recommended by participants at the CEBAF Workshop on the APT Design, April, 1977.

[4] R.S.Mills, K.R.Crandall, and J.A.Farrell, Proc. 1984 Linac Conf., Seeheim, Germany(May, 1984). 